\title{
Operative steps in management of benign nerve sheath tumors
}

\author{
Jonathon R. BALl, B.Med., B.Med.Sci., AND Michael T. Biggs, M.B.B.S., F.R.A.C.S. \\ Department of Neurosurgery, Royal North Shore and North Shore Private Hospitals, Sydney, \\ New South Wales, Australia
}

\begin{abstract}
$\checkmark$ Benign peripheral nerve sheath tumors include the neurofibroma, schwannoma, and their plexiform variants. Operative management begins with an assessment of the relative risks associated with surgery compared with observation. The risks of observation include the risk of malignancy, the progression of symptoms, risk of delayed surgery, and ongoing patient suffering. The risks of surgery include anesthetic problems, wound complications, and neurological injury. New neurological deficits have been reported to occur in approximately 10 to $15 \%$ of surgically treated cases. In general, surgery is recommended for symptomatic or progressive lesions. Although the surgical approach will vary depending on the location, type, and extent of tumor, adherence to certain principles will facilitate success. Adequate visualization and successful removal will be achieved with detailed anatomical knowledge, an adequate extensile exposure to visualize the proximal and distal tumor extent, circumferential dissection of the tumor, microsurgical dissection under appropriate magnification, and meticulous hemostasis throughout the procedure.
\end{abstract}

KEY WORDS - nerve sheath tumor - plexiform neurofibroma • schwannoma - surgery

$\mathrm{B}$ ENIGN PNSTs include the neurofibroma, schwannoma, and their plexiform variants. Although the exact details of surgical treatment will be dependent on the location and nature of the lesion, distinct operative steps can be defined. These can subsequently be individualized for a given situation. The importance of preoperative evaluation should be emphasized, including confirmation of the diagnosis and a risk-balanced approach to surgical decision making. Operative steps include appropriate incision, adequate exposure, detailed tumor inspection, microsurgical dissection, and tumor removal.

\section{Preoperative Considerations}

The initial step in disease management for a patient presenting with a benign PNST is to confirm the diagnosis by conducting a targeted history and examination, combined with the use of appropriate electrophysiological and imaging studies. ${ }^{7,8}$ In this process, attention should also be directed to the possible presence of a neurocutaneous syndrome. Percutaneous biopsy sampling should not form part of the workup, because biopsy procedures carry a risk of neurological injury, may seed tumor cells along the needle track, and cause scarring, which increases the challenge of definitive surgical removal.

\footnotetext{
Abbreviations used in this paper: $\mathrm{MR}=$ magnetic resonance; $\mathrm{NF} 1$ = neurofibromatosis Type $1 ; \mathrm{PNST}=$ peripheral nerve sheath tumor.
}

The decision to operate or observe will be based on the balance of the perceived risks of surgery against those associated with more conservative management, and will be tempered by the wishes of the patient.

\section{Risks of Observation}

The risks related to continued conservative management include the possibility of progressive neurological dysfunction and the presence or potential development of malignant change. Symptomatic lesions may cause ongoing suffering if left untreated, and some tumors may be cosmetically displeasing. Despite their frequency, data on the natural history of sporadic peripheral nerve tumors is lacking. There are no series reporting the incidence of neurological progression or malignant transformation in observed tumors, and no established guidelines exist on patient selection for surgery. In the general population, the incidence of malignant PNSTs is often quoted at $0.001 \%$, based on a poorly designed clinic-based study. ${ }^{3}$ In a large series from a specialist peripheral nerve surgical center, investigators reported that the incidence of malignancy in surgically treated tumors was $4 \%$ in patients without NF1 ${ }^{4}$ Interestingly, the majority of malignant tumors were not suspected to be so at the time of surgery. Nevertheless, they were more likely to be associated with pain and progressive neurological deficit. The overwhelming majority of these tumors occurred in the brachial plexus. The possibility of malignancy is greater in patients with a neurocutaneous syndrome. Rigorous epidemiological studies have estimated the lifetime risk to be 
between 8 and $13 \%$ in patients with NF1. ${ }^{4}$ The sizeable tumor burdens carried by sufferers preclude attempts at prophylactic resection of multiple lesions.

\section{Risks of Surgery}

Patients undergoing surgery for PNSTs are exposed to the risks of anesthesia, wound complications, and neurological injury. There is a significant incidence of neurological deterioration after PNST surgery. In a large published surgical series, Donner et al. ${ }^{2}$ reported an incidence of increased motor deficit in $11 \%$ of patients without NF1 who presented with weakness, and new motor deficits were found in $15 \%$ of patients without NF1 who had no weakness preoperatively. New painful sensory disturbances were reported in up to $10 \%$ of patients without NF1. These risks were significantly greater in the NF1 cohort. There are conflicting reports about whether neurofibromas carry a higher incidence of neurological injury compared with schwannomas. ${ }^{1,2}$ Preoperative counseling should include frank discussions about the magnitude of these risks and the potential occupational and personal implications.

Our general recommendation is for patients with PNSTs to undergo resection if the lesion is symptomatic or progressing in size. When evaluating tumors in locations associated with either a higher risk of malignancy (brachial plexus lesions) or a more significant risk of deficit (location in major nerve trunks), a lower threshold to operate may be appropriate.

\section{General Principles \\ Operative Considerations}

The overriding goal of the surgical management of benign nerve sheath tumors is to remove the lesion with minimal morbidity. Adherence to certain principles will facilitate success. Adequate visualization and successful removal will be achieved if the practitioner has detailed anatomical knowledge, achieves good exposure with meticulous hemostasis, and uses microsurgical techniques under appropriate magnification.

\section{Incision and Exposure}

An adequate, extensile exposure is essential for the effective removal of benign nerve sheath tumors. The healthy parts of the nerve of origin must be visualized proximally and distally to the lesion. For these purposes, small or transverse incisions are not appropriate. Incisions will generally follow the axial line of the extremities, except when crossing flexure creases, where a z-plasty may be most appropriate. Standard exposures for the major nerves and plexi have been well described. ${ }^{6}$ For tumors involving small cutaneous nerves, a linear incision directed along the path of the nerve will be suitable. The tumor and related nerve must be dissected circumferentially so that all surfaces are accessible and neighboring structures can be identified, dissected, and protected.

\section{Inspection of Tumor}

Before attempts at resection are commenced, the tumor and related nerve should be inspected visually and electrically. Identification of nerve fascicles over the surface of the tumor will allow safe planning of the intraneural dissec- tion. With the assistance of loupe magnification or the operating microscope, it is usually possible to appreciate the nerve fascicles splayed over the lesion. The functional state of these fascicles can be determined using electrical stimulation (discussed in a later section). By such an examination, the presence of fascicle-free tumor surface can be confirmed. It is through these areas that intraneural dissection can proceed.

\section{Intraneural Dissection}

The aim of this dissection is to separate the tumor and capsule from the uninvolved nerve fascicles. The initial incision is best positioned near the equator of the tumor and oriented parallel to the fibers of the nerve of origin. This minimizes the risk of injury to uninvolved nerve. The dissection most commonly proceeds in the extracapsular plane, by using a microdissector to tease the fascicles gently from the capsule. This separation proceeds toward the poles of the tumor. At the poles, fascicles will be encountered that enter the substance of the tumor. Electrical stimulation of these fascicles will help determine whether they are functional.

Classically, schwannomas are described as arising from a single fascicle, with the remainder of the nerve splayed over the surface. ${ }^{9}$ In contrast, multiple fascicles may be involved by the tumor mass of neurofibromas. Some authors have advocated intracapsular dissection in cases of adherent tumors, claiming that there is no increased risk of morbidity. ${ }^{10}$ When a larger tumor is encountered it may be necessary to debulk the lesion internally and allow the capsule to collapse inward to facilitate dissection. The use of an ultrasonic aspirator may be helpful in these circumstances. For proximal tumors arising from nerve roots exiting the spinal canal, only the distal portion is accessible. In these cases, a technique of asymmetrical tumor removal is used. ${ }^{10}$ The same principles of exposure and dissection are applied, but only to the accessible portion of nerve. Dissection proceeds in a retrograde fashion until the proximal end of the tumor is reached.

\section{Tumor Removal}

The remaining nerve fibers that enter or exit the tumor and are deemed to be nonfunctioning are sectioned. The lesion can then be removed from the tumor bed. After removal of the mass, the tumor bed is examined. A further confirmation of adequate hemostasis is sought and the wound is closed.

\section{Intraoperative Electrophysiological Tests}

The foregoing description of operative steps mentions the use of intraoperative electrophysiological techniques. Such techniques allow the evaluation of nerve fascicles involved with the tumor, distinguishing functional from nonfunctional nerves and therefore guiding the operative approach. ${ }^{7}$ Established options for nerve interrogation include the measurement of nerve action potentials or electromyographic responses to direct nerve simulation. In nerve sheath tumors in which access to the proximal segment may be limited (such as spinal foraminal tumors), motor evoked potentials to transcranial stimulation or sensory evoked responses to peripheral stimulation have been used. The availability of such techniques will vary among institutions, and special- 
ized staff and equipment are required. In the absence of sophisticated technology, the use of bipolar forceps connected to the anesthetist's peripheral nerve stimulator and set at an output of $2 \mathrm{~mA}$ and a frequency of $2 \mathrm{~Hz}$ (Stimlocator model SL3; B. Braun Pty. Ltd.) can stimulate the nerve, and direct observation of a motor response may provide valuable information. The decision to use a given method will also vary depending on the involved nerve and the experience of the surgeon. We would suggest that the use of these techniques is most beneficial when the surgeon's volume of cases is small. As surgical experience grows, lessons are learned from the use of intraoperative electrophysiological tests, the surgical technique is refined, and reliance on such technologies diminishes.

\section{Illustrative Cases}

\section{Case 1}

This 32-year-old woman presented with pain involving the posterior aspect of her thigh and calf when sitting. An MR image demonstrated the presence of an enhancing mass involving the sciatic nerve in the thigh (Fig. 1A). After consideration of the relative risks associated with observation compared with surgical treatment, her informed consent for tumor resection was obtained. At operation, tumor was identified arising from the tibial portion of the sciatic nerve (Fig. 1B). Nerve fascicles could be seen spread over the surface of the tumor. Surgical exposure was extended proximally and distally to normal nerve, the peroneal portion of the nerve was identified and protected, and the tibial portion was dissected free of its surroundings. After visual and electrical inspection of the tumor surface, inter-

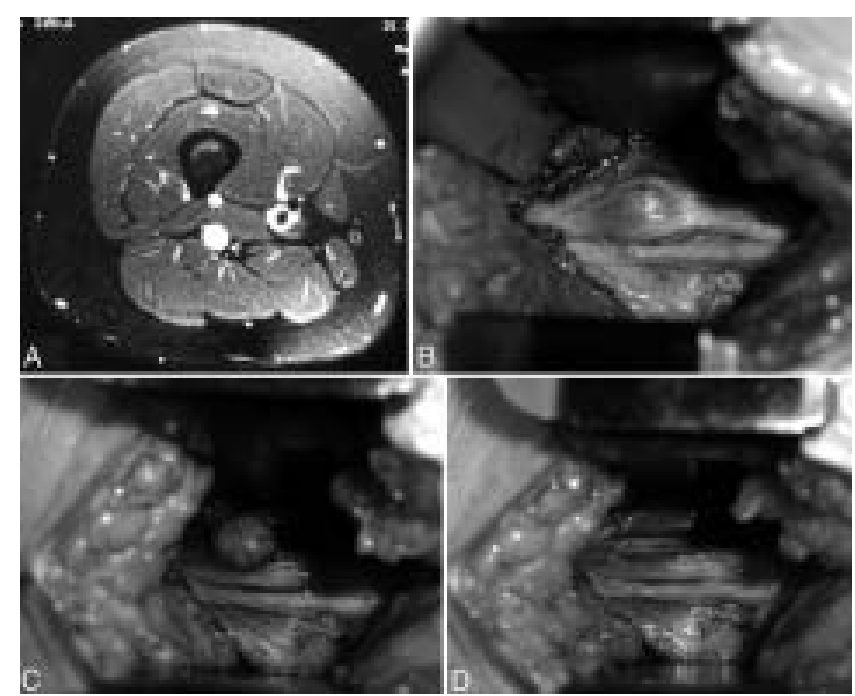

FIG. 1. CASE 1. Admission MR image and intraoperative photographs of a sciatic nerve schwannoma. The preoperative MR image demonstrates an enhancing lesion in the sciatic nerve (A). The operative steps of exposure and inspection (B), dissection (C), and removal (D) are illustrated in the photographs. The proximal part of the nerve is shown on the right side of the panels. The tumor was encountered in the tibial portion of the sciatic nerve, with fascicles stretched over the surface (B). Dissection revealed a single entering and exiting fascicle $(\mathrm{C})$. The tumor was removed, with preservation of the parent nerve (D). fascicular dissection was undertaken in the extracapsular plane (Fig. 1C). A single entering and exiting nerve fascicle were identified and found to be electrically silent. This was sectioned and the tumor removed as a whole (Fig. 1D).

\section{Case 2}

This 14-year-old girl presented with a 3-year history of a growing mass in her left upper limb associated with pain in the forearm. Minor weakness of finger and thumb extension was noted on examination. An MR image revealed an extensive enhancing mass arising from the posterior interosseous nerve (Fig. 2A). After consideration of the relative risks associated with observation compared with surgical treatment, informed consent for tumor resection was obtained. At operation, a multilobulated lesion was successfully resected, with preservation of the fascicular structure. No new neurological deficit was detectable postoperatively. Follow-up MR images obtained at 6 months postoperatively demonstrated recurrence of the lesion. Further imaging at 9 months demonstrated continued growth of the tumor (Fig. $2 \mathrm{~B}$ ), and a decision was made to perform a radical resection.

At the second operation, en bloc resection of the posterior interosseous nerve was performed from the wrist to just distal to the origin of the extensor carpi radialis longus branch. Postoperatively, finger and thumb extension was absent and useful wrist extension with radial deviation (Medical Research Council Score 4+/5) was preserved. After several months, tendon transfer from flexor carpi radialis to the four fingers and palmaris longus to the thumb was undertaken to restore extensor function to the digits. Two years after the second operation there was no tumor recurrence (Fig. 2C), and the patient had essentially normal hand function.

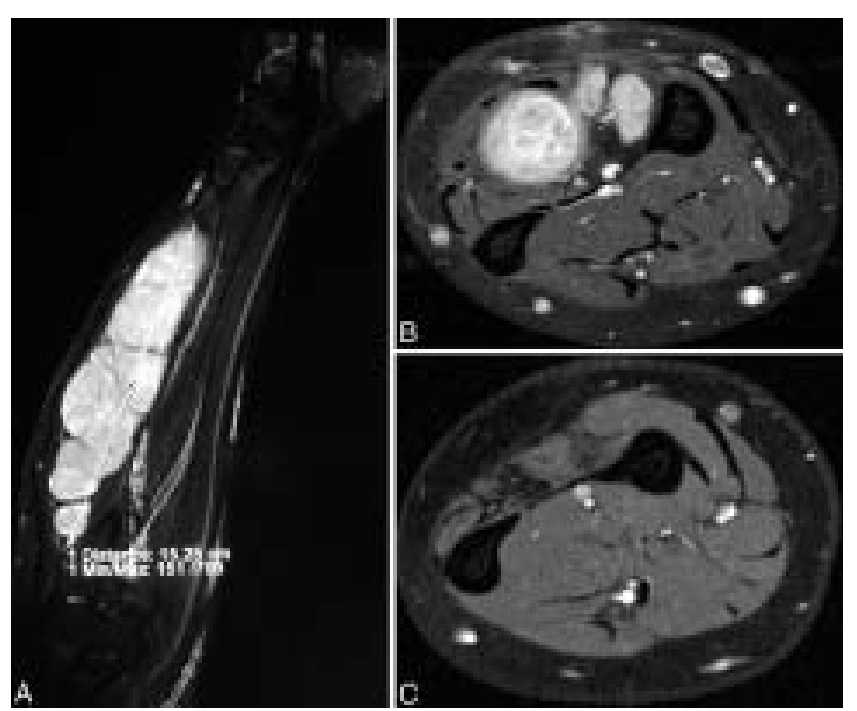

FIG. 2. CASE 2. Preoperative and follow-up MR images demonstrating posterior interosseous nerve plexiform schwannoma. A: The preoperative MR image demonstrates an enhancing lesion in the posterior interosseous nerve. B: Follow-up imaging performed 9 months postoperatively revealed extensive recurrence. Radical resection was undertaken and tendon transfer performed to restore hand function. C: Further imaging performed 2 years later demonstrated no evidence of recurrence. 


\section{Discussion}

These two cases demonstrate the application of the principles we have outlined and the flexibility required in surgical management of PNSTs. The first case involves a benign PNST in the patient's extremity. The operative steps were tailored to the location and nature of the tumor, and an excellent result was attained. The second case illustrates a situation in which a good neurological result was achieved but the tumor recurred. The extensive infiltrating nature of a plexiform tumor presents unique challenges; these lesions are associated with a greater chance of incomplete resection and neurological injury. ${ }^{1,5}$ Concomitantly, they are reported to have a higher incidence of malignant transformation. The involvement of a multidisciplinary surgical team and intensive rehabilitation can produce satisfactory results in such situations.

\section{Conclusions}

The surgical approach will vary according to the location, type, and extent of tumor. Once the diagnosis is established, the choice of management strategy will depend on an evaluation of relative risk. In general, symptomatic or progressive lesions are considered suitable for surgery. An extensile exposure with circumferential dissection of the tumor and nerve, both proximal and distal, must be obtained. The anatomy and function of associated nerve fascicles must be studied. An interfascicular approach allows dissection of the tumor. Flexibility in the application of these principles is necessary for tumors of the proximal nerve roots, larger tumors, or plexiform lesions for which a multidisciplinary surgical approach may be beneficial.

\section{References}

1. Artico M, Cervoni L, Wierzbicki V, D'Andrea V, Nucci F: Benign neural sheath tumors of major nerves: characteristics in 119 surgical cases. Acta Neurochir (Wien) 139:1108-1116, 1997

2. Donner TR, Voorhies RM, Kline DG: Neural sheath tumors of major nerves. J Neurosurg 81:362-373, 1994

3. Ducatman BS, Scheithauer BW, Piepgras DG, Reiman HM, Ilstrup DM: Malignant peripheral nerve sheath tumors. A clinicopathologic study of 120 cases. Cancer 57:2006-2021, 1986

4. Evans DG, Baser ME, McGaughran J, Sharif S, Howard E, Moran A: Malignant peripheral nerve sheath tumors in neurofibromatosis 1. J Med Genet 39:311-314, 2002

5. Kim DH, Murovic JA, Tiel RL, Moes G, Kline DG: A series of 397 peripheral neural sheath tumors: 30 -year experience at Louisiana State University Health Sciences Center. J Neurosurg 102:246-255, 2005

6. Kline DG, Hudson AR, Kim DH: Atlas of Peripheral Nerve Surgery, ed 2. Philadelphia: WB Saunders, 2001

7. Maniker A: Diagnostic steps, imaging, and electrophysiology. Neurosurg Clin N Am 15:133-144, 2004

8. Ramcharan R, Midha R: Clinical presentation and physical examination. Neurosurg Clin N Am 15:125-132, 2004

9. Skovronsky DM, Oberholtzer JC: Pathologic classification of peripheral nerve tumors. Neurosurg Clin N Am 15:157-166, 2004

10. Tiel R, Kline D: Peripheral nerve tumors: surgical principles, approaches, and techniques. Neurosurg Clin N Am 15:167-175, 2004

\section{Manuscript submitted April 2, 2007.}

Accepted April 25, 2007.

Address reprint requests to: Michael T. Biggs, M.B.B.S., F.R.A.C.S., North Shore Neurosurgery, Suite 5, Level 6, North Shore Medical Centre, 66-80 Pacific Highway, St. Leonards, Sydney, New South Wales 2065, Australia. email: biggs@idx.com.au. 